reprint: IEEE Transactions on Intelligent Transportation Systems, Vol. 1, No. 4, December 2000, pp. 179-189.

\title{
A Review of Conflict Detection and Resolution Modeling Methods
}

\author{
James K. Kuchar and Lee C. Yang \\ Massachusetts Institute of Technology \\ Cambridge, MA 02139 USA
}

\begin{abstract}
A number of methods have been proposed to automate air traffic conflict detection and resolution (CD\&R), but there has been little cohesive discussion or comparative evaluation of approaches. This paper presents a survey of 68 recent CD\&R modeling methods, several of which are currently in use or under operational evaluation. A framework that articulates the basic functions of $C D \& R$ is used to categorize the models. The taxonomy includes: dimensions of state information (vertical, horizontal, or three-dimensional); method of dynamic state propagation (nominal, worst-case, or probabilistic); conflict detection threshold; conflict resolution method (prescribed, optimized, force field, or manual); maneuvering dimensions (speed change, lateral, vertical, or combined maneuvers); and management of multiple aircraft conflicts (pairwise or global). An overview of important considerations for these and other CD\&R functions is provided, and the current system design process is critiqued.
\end{abstract}

\section{INDEXTERMS}

Conflict detection and resolution, air traffic control, alerting systems, warning systems.

\section{INTRODUCTION}

Methods for maintaining separation between aircraft in the current airspace system have been built from a foundation of structured routes and evolved procedures. Humans are an essential element in this process due to their ability to integrate information and make judgments. However, because failures and operational errors can occur, automated systems have begun to appear both in the cockpit and on the ground to provide decision support and to serve as traffic conflict alerting systems. These systems use sensor data to predict conflicts between aircraft and alert humans to a conflict, and may provide commands or guidance to resolve the conflict. Relatively simple conflict 
predictors have been a part of air traffic control automation for several years, and the Traffic Alert and Collision Avoidance System (TCAS) has been in place onboard domestic transport aircraft since the early 1990s (Figure 1). Together, these automated systems provide a safety net should normal procedures and controller and pilot actions fail to keep aircraft separated beyond established minimums.

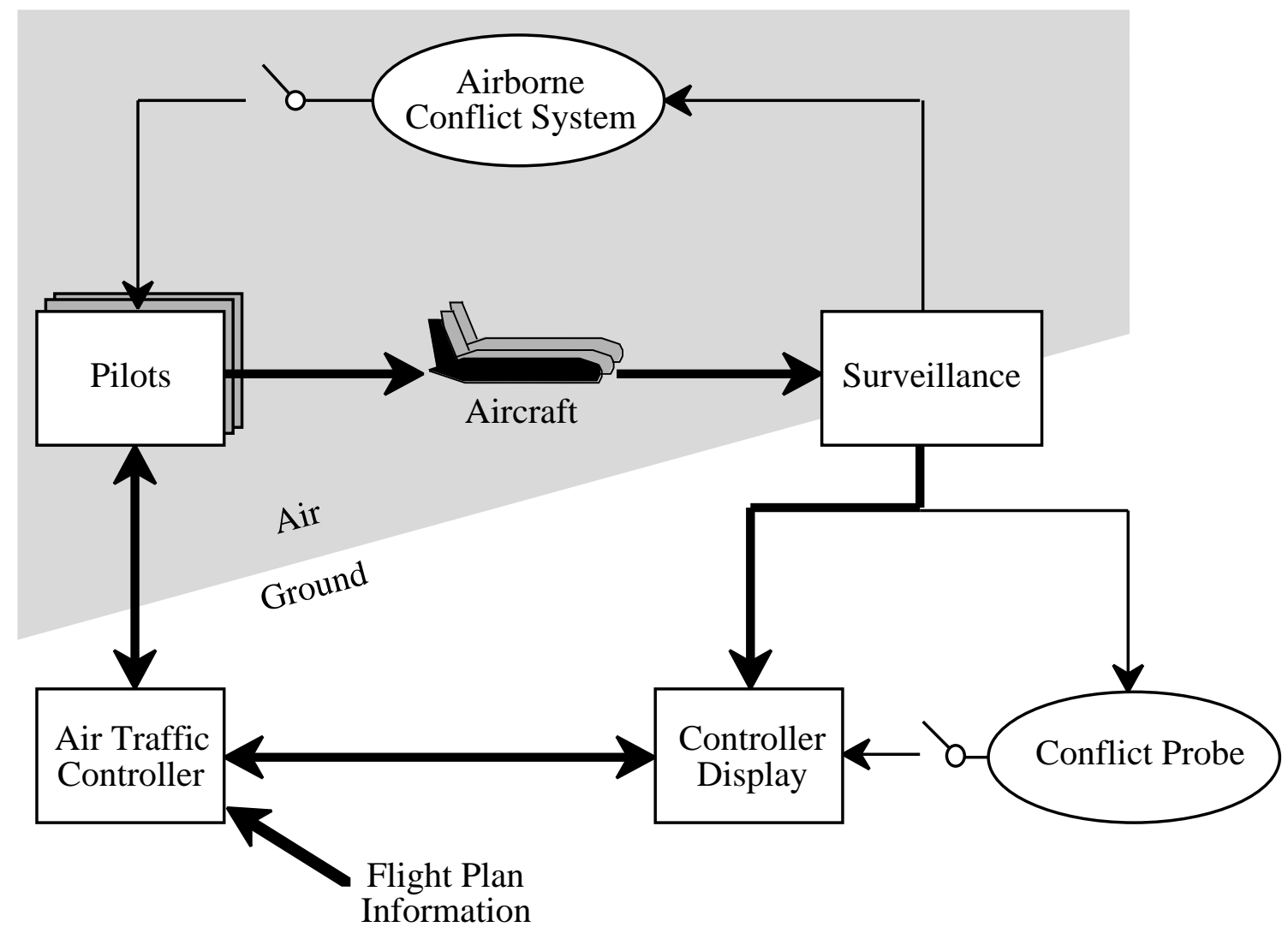

Fig. 1. Air and ground components of conflict detection and resolution (bold lines represent the nominal control path, thin lines represent automated monitoring)

Recently, interest has grown toward developing more advanced automation tools to detect traffic conflicts and assist in their resolution. These tools could make use of future technologies, such as a data link of current aircraft flight plan information, to enhance safety and enable new procedures to improve traffic flow efficiency. For example, several concepts for automation to aid air traffic controllers have been studied and implemented in the field, including components of the 
Center / TRACON Automation System (CTAS) developed by NASA [1], and the User Request Evaluation Tool (URET) developed by MITRE [2]. Advanced airborne conflict detection and resolution (CD\&R) systems are also under study as more strategic alternatives to TCAS [3]. With the growth of airspace congestion, there is an emerging need to implement these types of tools to assist the human operators in handling the expanding traffic loads and improve flow efficiency.

In total, over 60 different methods have been proposed by various researchers to address CD\&R. These methods have been developed not only for aerospace, but also for ground vehicle, robotics, and maritime applications, because the fundamental conflict avoidance issues are similar across transportation modes. A review of recent CD\&R research suggests that the current environment is one in which a given solution approach to the problem is proposed and exercised, typically through a set of constrained and simplified examples. There has been little crosscutting comparison or synthesis between methods. The result is a jumble of models, each with its own champion, with little structure in which to understand and relate concepts. Thus, there is a distinct need for a framework that can be used to compare, contrast, and evaluate CD\&R methods.

Some initial steps toward describing and understanding the differences and similarities between CD\&R models have recently occurred. Zeghal [4], for example, provides a review of the major differences among so-called force field methods for conflict resolution, and Warren [5] conducted a comparative evaluation between three conflict detection methods. Additionally, Krozel et al. [6] and Kuchar \& Yang [7] previously conducted initial surveys of current methods in CD\&R.

In response to the need for an updated and broader deliberation of the modeling issues, this paper provides a summary and discussion of the major approaches from the recent literature that have been used to address CD\&R problems. The intent is not to recommend any given model, since each requires considerably more analysis than can be conducted here. Rather, the intent is to create a taxonomy in which to place a given model, point out its advantages and disadvantages, and identify common issues that should be considered in future development and evaluation studies. 
The goal is that this framework may be of use when developing new models or when performing comparisons between models.

\section{CONFlict Detection AND Resolution Processes}

To begin, it is necessary to have a clear definition of a conflict. In this framework, a conflict is an event in which two or more aircraft experience a loss of minimum separation. In other words, the distance between aircraft violates a criterion defining what is considered undesirable. One example criterion is a minimum of $5 \mathrm{nmi}$ of horizontal distance between aircraft or at least $1000 \mathrm{ft}$ of vertical separation (the current en-route separation standard at lower altitudes). The result is a protected zone (PZ) or volume of airspace surrounding each aircraft that should not be infringed upon by another vehicle. The PZ could also be defined as a much smaller region (e.g., a sphere $500 \mathrm{ft}$ in diameter) in the case of tactical collision alerting systems, or even in terms of parameters other than distance (e.g., time). In any case, the underlying CD\&R functions are similar, although the specific models and alerting thresholds would likely be different.

The goal for the CD\&R system is to predict that a conflict is going to occur in the future, communicate the detected conflict to a human operator, and, in some cases, assist in the resolution of the conflict situation. These three fundamental processes can be organized into several phases or elements as shown in Figure 2. Conflicts with hazards other than another aircraft can be abstracted to the same fundamental decision-making problem. Accordingly, terrain proximity warning systems are also included in the discussion here, and systems to warn of other hazards (such as weather) could be included as well.

As shown in Figure 2, the traffic environment must first be monitored and appropriate current state information must be collected and disseminated using sensors and communications equipment. These states provide an estimate of the current traffic situation (e.g., aircraft position and velocity). Because of the types of sensors that are used, these states may not completely describe the actual situation. For example, a system may only have access to range information 
between aircraft, and be unable to determine bearing. Additionally, due to sensor errors or limited update rate, there is generally some uncertainty in the values of the current states that are available.

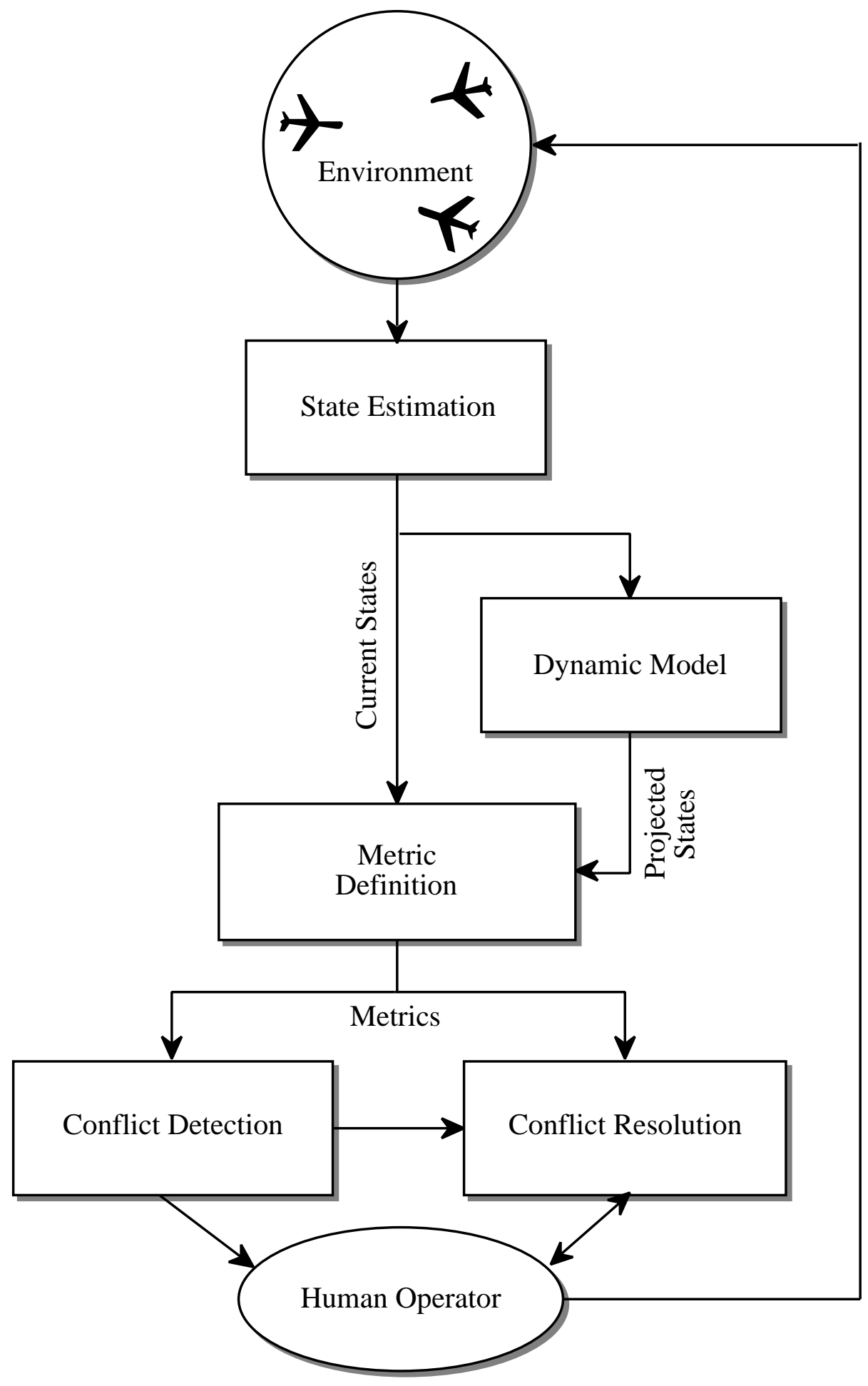

Fig. 2. Conflict detection and resolution processes 
A dynamic trajectory model is also required to project the states into the future in order to predict whether a conflict will occur. This projection may be based solely on current state information (e.g., a straight-line extrapolation of the current velocity vector) or may be based on additional, procedural information such as a flight plan. As with the current state information, there is generally some uncertainty in the estimate of the future trajectory.

Information regarding the current and predicted states can then be combined to derive metrics used to make traffic management decisions. Some example metrics include predicted minimum separation or the estimated time to closest point of approach. Whereas the current and projected states can generally be estimated independently for each aircraft, the conflict metrics require some form of aggregation of the states between the different vehicles involved.

Given the conflict metrics, a discrete decision (Conflict Detection) is then made regarding whether a human should be informed and whether action is needed to maintain traffic separation. In some cases, notification of a conflict is all that is required of the CD\&R system; the human operator then determines how to resolve the conflict safely and efficiently. Note, however, that not all predicted conflicts require notification or action. For example, a conflict may be predicted to occur, but be far enough into the future or uncertain enough that an alert would be a nuisance and action would not be appropriate at the current time. For the purposes of this paper, a conflict is detected once it is both predicted to occur and it has been determined that it is appropriate to alert the operator.

When action is considered necessary, the Conflict Resolution phase may be initiated. This involves determining an appropriate course of action and transmitting that information to the operators. For example, TCAS issues Resolution Advisories to the pilot that command a target rate of climb or descent to avoid a collision. Other methods may be more passive and simply provide feedback to the operator about whether a manually entered trial action will resolve the conflict. Although the conflict resolution phase is shown as a single block in Figure 2, it requires its own 
set of current state estimates, a resolution maneuver trajectory model, and decision criteria which may be different from those used in the Conflict Detection phase.

Either or both Conflict Detection and Conflict Resolution may be automated or may be handled manually through procedures. For example, Visual Flight Rules (VFR) place the responsibility for collision avoidance on the pilot, who must visually scan for traffic (conflict detection) and if a threat is perceived, take appropriate action according to a set of "rules of the road" (conflict resolution). Under Instrument Flight Rules (IFR), an Air Traffic Controller monitors traffic separation using radar and issues vectors to aircraft when a conflict is projected to occur. If conflicts are not resolved by the human operators themselves, resolution information is automatically issued by TCAS to provide additional guidance.

In this framework, conflict detection can be thought of as the process of deciding when action should be taken, and conflict resolution involves determining how or what action should be performed. In practice, however, it is not always clear how to separate conflict detection from conflict resolution. For example, deciding when action is required may depend on the type of action that will be performed. Similarly, the type of action that is required may depend on how early that action begins. This interdependence is one factor that makes CD\&R system development challenging and interesting, because there are many feasible design solutions.

\section{CATEGORIZATION OF MODELING APPROACHES}

To provide insight into different methods of conflict detection and resolution, a literature review of previous research models and current developmental and operational systems was performed. The overall modeling approaches from a total of 68 methods or systems are discussed here. These models do not represent an exhaustive list, but are believed to encompass the major recent approaches to $C D \& R$ problems. Several authors have proposed more than one CD\&R method in a single publication; these cases are listed separately where appropriate. Additionally, some effort is underway investigating the human factors issues associated with conflict detection 
and resolution $[8,9]$. However, this paper is directed only at numerical or analytical models for evaluating traffic conflict scenarios, not at human-centered issues.

Nine of the models that were examined are existing operational systems in use or which have been evaluated in the field: Airborne Information for Lateral Spacing (AILS) [10], CTAS [1], Ground Proximity Warning System (GPWS) [11] and its recent Enhanced version (EGPWS) [12], Precision Runway Monitor (PRM) [13], TCAS [14], Traffic and Collision Alert Device (TCAD) [15], URET [2], and a prototype conflict detection system for the Cargo Airline Association [3]. The remaining models range from abstract concepts to prototype conflict warning systems being evaluated or used in laboratories. Five of the models were developed for robotic, automobile, or naval applications [16-20], but are still applicable to aviation.

Based on the framework in Figure 2, the 68 models were catalogued according to their fundamental approaches to each phase of the CD\&R process. To provide a consistent basis upon which to describe the models, each model is classified by the manner in which it is explicitly described in its reference. A model defined here to address only horizontal conflicts, for instance, could potentially be extended to work in three dimensions (and the need for such an extension may have been mentioned in the reference), but such an extension was not specifically described in the reference. As another example, if a model computes aircraft miss distance but does not define an explicit conflict detection threshold, the model is not classified as providing Conflict Detection even though the model could be adapted to perform such a task.

The 68 models are organized in terms of six key design factors, discussed in more detail below. Although other differences between the models exist that are not covered here, the six factors that are used represent principal categories by which models differ. An important caveat is that the models have been analyzed, verified, and validated to varying degrees by their developers and the community. This paper focuses only on the specific attributes of each model, not on the depth to which a model has been analyzed, validated, or accepted. Thus, care should be taken to remember that a model that seems to be simple according to our categorization scheme may be 
significantly more viable than an apparently sophisticated model. Similarly, two models may be identical in terms of the six design factors used here but may be quite different in terms of implementation or maturity. More detail on 33 of the 68 models covered here is available in Kuchar \& Yang [7].

\section{A. State Propagation}

Because conflict detection and resolution can only be as reliable as the ability of the model to predict the future, the most concrete difference between modeling approaches involves the method by which the current states are projected into the future. Three fundamental extrapolation methods have been identified, termed Nominal, Worst-case, and Probabilistic. The three methods are shown schematically in Figure 3.

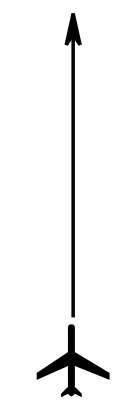

Nominal

(a)

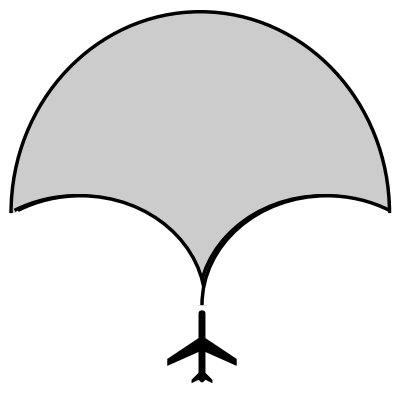

Worst-case

(b)

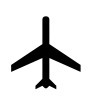

Probabilistic

(c)

Fig. 3. State propagation methods

In the Nominal method, the current states are projected into the future along a single trajectory, without direct consideration of uncertainties. An example would be extrapolating the aircraft's position based on its current velocity vector (Figure 3a). The Nominal projection method is straightforward, and provides a best estimate of where the aircraft will be, based on the current state information. In situations in which aircraft trajectories are very predictable (such as when 
projecting only a few seconds into the future), a Nominal trajectory model may be quite accurate. Nominal projections, however, do not directly account for the possibility that an aircraft may not behave as expected - a factor that is especially important in longer-term conflict detection. Generally, this uncertainty is managed by introducing a safety buffer, minimum miss distance, or time to closest point of approach threshold at which point a conflict will be detected. Alerts for conflicts which are predicted to occur far in the future using a Nominal trajectory model will need to be inhibited so as to not cause a nuisance to the operator.

The other extreme of dynamic modeling is to examine a Worst-case projection. Here, it is assumed that an aircraft will perform any of a range of maneuvers. If any one of these maneuvers could cause a conflict, then a conflict is predicted. The result is a swath of potential trajectories which is monitored to detect conflicts with other aircraft (Figure 3b). Worst-case approaches are conservative in that they can trigger conflict alerts whenever there is any possibility of a conflict within the definition of the Worst-case trajectory model. If such conflict-inducing maneuvers are unlikely, protecting against them may severely reduce overall traffic capacity due to a high false alarm rate. Accordingly, the Worst-case trajectory must be limited to a certain look-ahead projection time. Still, the Worst-case approach may be appropriate when it is desirable to determine if a conflict is possible, or for air traffic concepts in which aircraft are procedurally constrained to remain within a given maneuvering corridor. Each corridor then becomes the boundary of the Worst-case aircraft trajectories, and conflicts can be predicted based simply on whether corridors intersect at the same point in time.

In the Probabilistic method, uncertainties are modeled to describe potential variations in the future trajectory of the aircraft (Figure 3c). This is usually done in one of two ways. In URET and CTAS, for example, a position error is added to a nominal trajectory, from which the conflict probability can be derived [1,2]. A second approach is to develop a complete set of possible future trajectories, each weighted by a probability of occurring (e.g., using probability density functions). The trajectories are then propagated into the future to determine the probability of conflict. 
A Probabilistic approach provides an opportunity for a balance between relying on either a single-trajectory model or a set of worst-case maneuvers. The advantage of a Probabilistic approach is that decisions can be made on the fundamental likelihood of a conflict; safety and false alarm rate can be assessed and considered directly. The Probabilistic method is also the most general: the Nominal and Worst-case models are subsets of Probabilistic trajectories. The Nominal trajectory corresponds to a case in which the aircraft will follow a given (maximum likelihood) trajectory with probability one; the Worst-case model is one in which the aircraft will follow any trajectory with equal likelihood. However, the logic behind a probability-based system may be difficult to convey to operators, possibly reducing their confidence [21]. There may also be difficulty in modeling the probabilities with which future trajectories will be followed.

Tables 1, 2, and 3 provide an organized listing of the 68 models. To conserve space, only the first author is listed in cases where there are multiple authors on a publication. The three Tables include those models using Nominal, Worst-case, or Probabilistic state propagation, respectively. Within each table, the models are organized according to the approach that each takes across several stages in CD\&R from Figure 2. Five columns are used to organize the models: State Dimensions, Conflict Detection, Conflict Resolution, Resolution Maneuvers, and Multiple Conflicts, each of which is described below and summarized in Table 4.

\section{B. State Dimensions}

The Dimensions column shows whether the state information used in the model involves purely the horizontal plane $(\mathrm{H})$, vertical plane $(\mathrm{V})$, or both $(\mathrm{HV})$. The majority of models cover either three-dimensions or the horizontal plane; only GPWS focuses solely on the vertical plane. Some models may be easily extended to cover additional dimensions than are shown here, but such extension is not explicitly described in the reference. It also must be noted that coverage of a certain dimension does not necessarily mean that a complete description of the situation in that dimension is available. For example, TCAS uses range measurements and range-rate estimates to determine if a conflict exists in the horizontal plane. A better prediction of the threat condition 
could be obtained if additional information were available, such as relative bearing. Ultimately, one would like to have a full four-dimensional description of the aircraft trajectories over time. The lack of complete observability of the conflict situation can lead to false alarms or late (or missed) detection events.

\section{Conflict Detection}

The Detection column indicates (with a check mark) whether each model explicitly defines when a conflict alert is issued. Models that do not have this explicit threshold may provide valuable, detailed tools and metrics upon which conflict detection decisions can be made, but do not explicitly draw the line between predicted conflict and non-conflict. Additionally, models shown to not provide conflict detection may be primarily concerned with the resolution of a conflict rather than in determining when that resolution should begin. Although developing conflict resolution methods is important, at some point it will be necessary to define conflict detection thresholds and examine the false alarm / missed detection tradeoffs. Models that are shown to provide conflict detection may use an extremely simple criterion (e.g., current range) to determine when a conflict exists or may use a more complex threshold or set of logic.

\section{Conflict Resolution}

The Resolution column shows the method by which a solution to a conflict is generated. Five categories are included here: Prescribed (P), Optimized (O), Force field (F), Manual (M), and no resolution (-).

Prescribed resolution maneuvers are fixed during system design based on a set of predefined procedures. For example, GPWS issues a standard "Pull Up" warning when a conflict with terrain exists. GPWS does not perform additional computation to determine an optimal escape maneuver. AILS [10] and Carpenter \& Kuchar [22] assume that a fixed climbing-turn maneuver is always performed to avoid traffic on a parallel runway approach. Prescribed maneuvers may have the benefit that operators can be trained to perform them reflexively. This may decrease response 
time when a conflict alert is issued. However, prescribed maneuvers are less effective, in general, than maneuvers that are computed in real time since there is no opportunity to modify the resolution maneuver - the maneuver is performed open-loop to some extent. In many conflicts, it will be necessary to adapt the resolution maneuver to account for unexpected events in the environment, or to reduce the aggressiveness of the maneuver should the conflict be resolved more easily than first predicted.

Optimization approaches typically combine a kinematic model with a set of cost metrics. An optimal resolution strategy is then determined by solving for the trajectories with the lowest cost. TCAS, for example, searches through a set of potential climb or descent maneuvers and selects the least-aggressive maneuver that still provides adequate protection [14]. This requires the definition of appropriate cost functions - typically projected separation, or fuel or time, but costs could also cover workload. Developing costs may be fairly straightforward for economic values but difficult when modeling subjective human utilities. Because current interest in this field is generally centered on strategic resolution of conflicts before immediate tactical evasion is required, economic costs and operator workload will be important to the system design.

Some of the models denoted as using Optimized conflict resolution apply techniques such as game theory, genetic algorithms, expert systems, or fuzzy control to the problem. Expert system methods use rule bases to categorize conflicts and decide whether to alert and/or resolve a conflict. These models can be complex and would require a large number of rules to completely cover all possible encounter situations. Additionally, it may be difficult to certify that the system will always operate as intended, and the "experts" used to develop or train the system may in fact not use the best strategy in resolving conflicts. However, a rule base, by design, may be easier for a human to understand or explain than an abstract mathematical algorithm.

Force field approaches treat each aircraft as a charged particle and use modified electrostatic equations to generate resolution maneuvers. The repulsive forces between aircraft are used to define the maneuver each performs to avoid a collision. A force field method, while attractive in the 
sense that a conflict resolution solution is continuously available using relatively simple equations, may have some pathologies that require additional consideration before they can be used in operation. For example, force field methods may assume that aircraft continuously maneuver in response to the changing force field, or that aircraft can vary their speed over a wide range. This requires a high level of guidance on the flight deck and increases complexity beyond issuing simple heading vectors, for example. Additionally, sharp discontinuities in the commanded resolution maneuvers may occur that require additional processing or filtering to arrive at physically feasible solutions. Several human-in-the-loop implementations of the force field method, however, appear to have resolved these problems and have shown that force field resolution can be effective when properly applied [23-25].

Some models allow the user to generate potential conflict resolution solutions and obtain feedback as to whether the trial solution is acceptable. These models are denoted as handling a Manual solution in the table. The benefit of a manual solution is that it is generally more flexible in the sense that it is based on human intuition, using information that may not be available to the automation. For example, weather information that is not available to the CD\&R system may be important when considering a conflict resolution maneuver. Automated solutions that do not take relevant environmental information into account will likely produce nuisance solutions that the human finds unacceptable.

A "_." in the Resolution column indicates that the model does not provide an explicit output of an avoidance action or feedback on a user-defined trial solution. These models perform conflict detection but are not designed to explicitly consider conflict resolution. In some cases, successful conflict resolution is presumed - the focus of the model is only on detecting or counting conflicts.

\section{E. Resolution Maneuvers}

The Maneuvers column indicates what dimensions of resolution maneuvers are allowed. Possible maneuver dimensions include Turns (T), Vertical maneuvers (V), and Speed changes (S). 
The notation TV, for example, means that either turns or vertical maneuvers may be performed (but not both simultaneously). In some cases, combined maneuvers may be commanded or performed, indicated by $\mathrm{C}($ ). Thus, $\mathrm{C}(\mathrm{TV})$, for example, indicates that a simultaneous climbing or descending turn may be performed.

Generally, providing more maneuvering dimensions allows for a more efficient solution to a conflict. However, it does place additional liens on the operator in the sense that a more complex maneuver must be controlled and monitored, possibly increasing response time and workload.

\section{F. Multiple Conflicts}

Finally, the Multiple column describes how the model handles situations with more than two aircraft. This can take two forms: Pairwise (P), in which multiple potential conflicts are resolved sequentially in pairs; and Global $(\mathrm{G})$, in which the entire traffic situation is examined simultaneously.

In a realistic traffic environment, it will be necessary that a CD\&R system be able to manage encounters involving more than two aircraft. In a pairwise approach, if one conflict solution induces a new conflict, the original solution may be modified until a conflict-free solution is found. This is the approach taken by TCAS, for example, and is effective but also could potentially fail in certain situations. A global solution that considers more than one other aircraft at a time, while more complex, may be more robust. For example, consider the situation shown in Figure 4. On the left, a pairwise solution is shown. The aircraft on the left detects a conflict with a coaltitude threat at a certain preset time before collision, and attempts to climb or descend. Neither solution is acceptable since it results in a conflict with another aircraft. On the right, a global solution considers all three threat aircraft simultaneously and determines that the climbing or descending maneuver must begin earlier than the baseline threshold time in order to safely resolve the conflict. At the least, models should be examined in multi-aircraft situations to determine their robustness to this type of problem. 


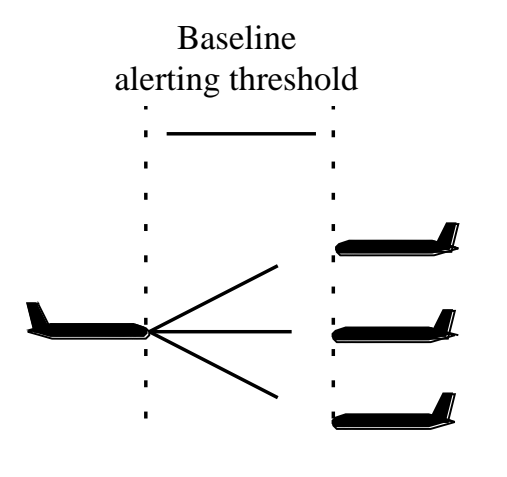

Pairwise Solution

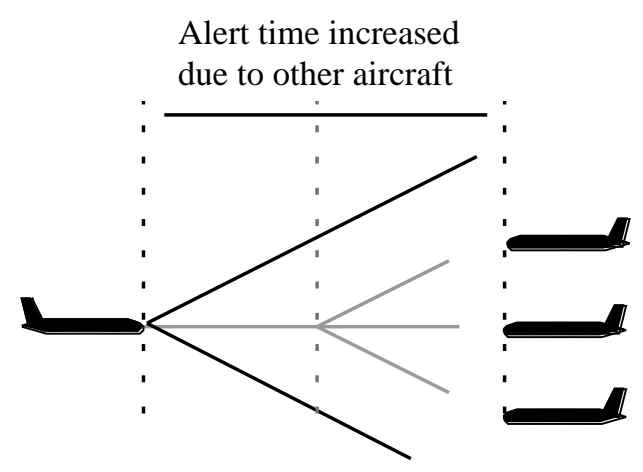

Global Solution

Fig. 4. Multiple aircraft conflict detection and resolution

\section{G. Other Model Elements}

In addition to the six factors used to distinguish between modeling approaches in Tables 13 , there are several other issues to be considered. These issues include specifically which current states and metrics are used to make CD\&R decisions, how uncertainty is managed in the model, and the degree to which the model assumes coordination between aircraft involved in a conflict. A number of the models surveyed here do address one or more of these issues, but they cannot be described fully here due to space limitations.

Consideration of the states that are used in CD\&R is important because these states represent the means by which the system observes the environment. Some CD\&R methods use a simplified set of states, which reduces sensor requirements but decreases the certainty with which conflict detection or resolution decisions can be made. Including aircraft intent information beyond the current position and velocity vector (e.g., a programmed flight plan) will be valuable in strategic conflict detection. Intent information can be used to better model the future trajectory of the aircraft, and thereby make more correct alerting decisions.

The manner in which uncertainties are managed in the design of a CD\&R system varies widely. Most approaches to the problem combine the uncertainties into a spatial safety buffer to 
reduce missed detection probability and also incorporate a look-ahead time limit to limit false alarms. This provides for a reasonable accommodation of uncertainty, but it may not be as effective or accurate as more complete, probabilistic trajectory models.

Coordinating conflict resolution between aircraft has two primary benefits. First, the required magnitude of maneuvering for a given aircraft may be reduced when two aircraft maneuver cooperatively when compared against a case in which only one aircraft maneuvers. Second, coordination helps ensure that aircraft do not maneuver in a direction that could prolong or intensify the conflict. However, coordination may increase controller or pilot workload due to the need to monitor several changes in the air traffic situation at one time. In any case, a system designed assuming that coordination will occur should also be evaluated in cases in which coordination is not carried out as planned. This would provide some measure of the robustness of the system to a data link failure or human error.

\section{SYSTEM DESIGN PROCESS ISSUES}

The surprising aspect to all of these modeling efforts is that no single solution has stood out as being clearly the most efficient or effective. A closer examination of the CD\&R system design process can help uncover the underlying principles that impact performance. The fundamental approach that has been used to date in the majority of cases to design and evaluate CD\&R systems involves the process shown schematically in Figure 5. First, a dynamic (and typically deterministic) trajectory model and set of alerting threshold metrics are developed for the CD\&R system, often based on engineering intuition. A typical example is that aircraft are assumed to fly in straight lines, and that time to minimum separation is a reasonable alerting threshold metric. This model and its parameters (e.g., the threshold time settings) are then exercised in a series of simulations (either through fast-time Monte Carlo simulation or human-in-the-loop studies). The alerting logic is exposed to a wide range of encounter situations, and the resulting number of false alarms and loss-of-separation events (or other statistical performance metrics) are recorded [2628]. Example situations typically include a variety of conflict geometries and aircraft dynamic 
behavior. This allows for uncertainties to be modeled and injected into the design of the system in order to explore system performance and robustness to uncertainty. If the observed system performance does not meet design specifications, then the model or the alerting thresholds are modified. For example, time or range thresholds are successively modified until there is an acceptable balance between loss of separation incidents and false alarms over the set of test scenarios. The result can be a complex, iteratively-evolved set of logic and threshold definitions. The TCAS alerting thresholds, for instance, have numerous kinks and overhead associated with special cases using if-then logic [14].

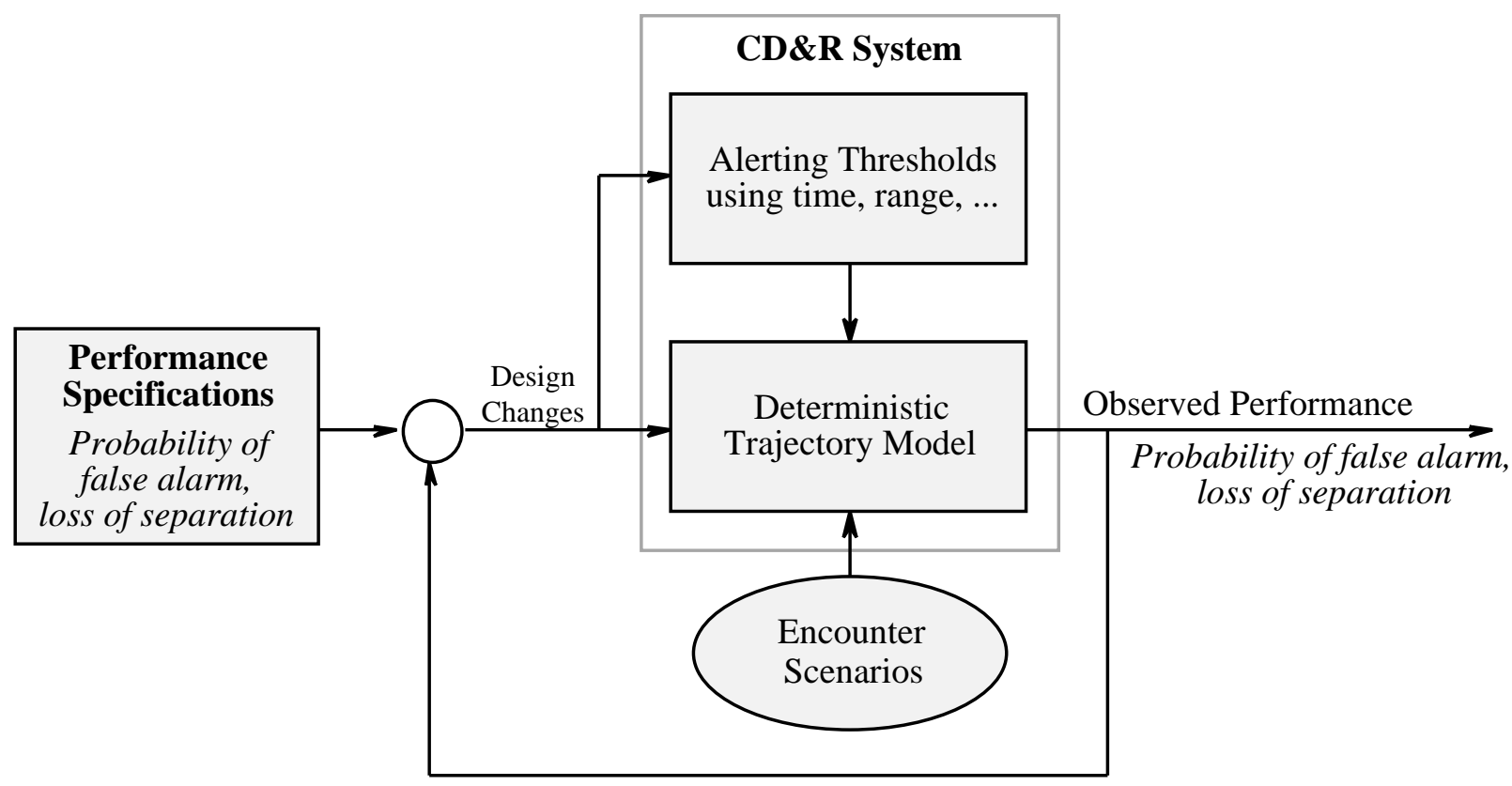

Fig. 5. Typical CD\&R system design and evaluation process

Ultimately, it is the observed performance in terms of false alarms and frequency of loss of separation that determines whether a system design is acceptable. In more strategic cases, economic or other costs can also be included. Closer examination of the CD\&R design method in Figure 5 reveals that, at its core, what is happening is that the parameters of the system are being tuned to the situations that are provided in the evaluation simulations. A change in the mix of encounter situations could lead to a change in the trajectory models, metrics, or threshold settings 
in order to meet desired performance constraints. The process is somewhat analogous to designing a control system compensator, but in this case the CD\&R system is essentially mapping the given encounter situations into false alarm rate or separation performance. In a sense, metrics such as range, miss distance, or time are simply surrogates for the real metrics - statistical measures of performance. A variety of different decision metrics could be used to achieve the same overall system performance, as reflected by the diversity of models reviewed here. Also, it is important to note that the mix of encounter situations that are used to exercise the CD\&R system in fact form a probabilistic or statistical model of the environment. To obtain the most accurate estimate of system performance, this statistical model should match the actual environment as closely as possible.

The surrogate system parameters that are developed through the process in Figure 5 are essentially global solutions averaged over the range of encounter situations that are tested. Due to the averaging process, there may be certain encounters in which the system performance could be improved. If this deficiency is significant, the CD\&R logic can be broken down into submodels, with varying dynamic models or thresholds in order to better manage the problem cases. In TCAS, for example, alerting threshold parameter values vary across several strata of altitude to better account for uncertainties in altitude measurement and changes in aircraft performance [14]. Ideally, the system should continuously tailor the dynamic model and the alert thresholds to the specific situation that it is monitoring.

A more direct approach to system design would be to use the information contained in the simulation and evaluation scenarios to build a probabilistic trajectory model for use by the CD\&R system (Figure 6). Since it is in the designer's best interest to use the most accurate encounter model that is possible during evaluation, this same model would contain the best information describing the environment in which the $C D \& R$ system will operate. Because it is using a probabilistic trajectory model, the $\mathrm{CD} \& \mathrm{R}$ system would also be able to estimate performance metrics such as probabilities of false alarm or loss of separation in real time. Then, rather than making a decision based on an indirect metric such as time to minimum separation, the alerting 
decision can be based on a direct comparison of the computed false alarm or loss of separation probabilities against the desired performance specifications.

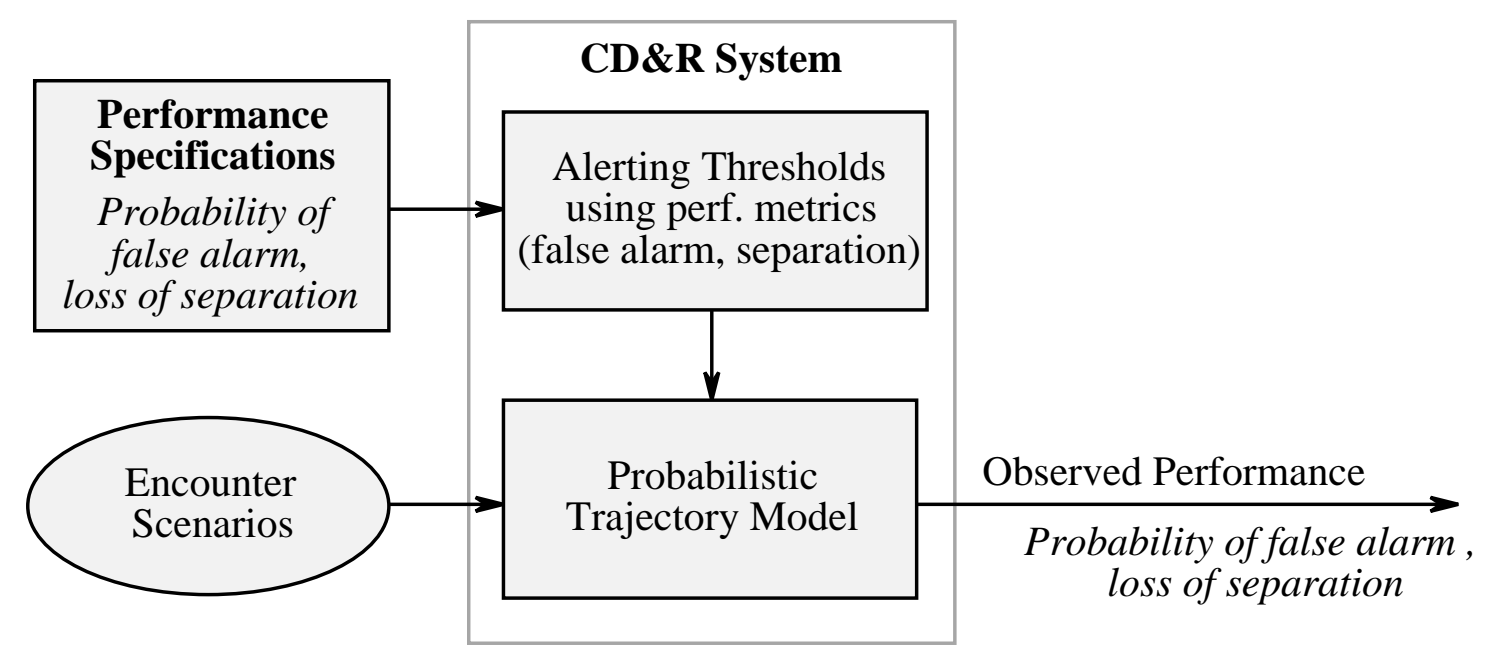

Fig. 6. Performance-based CD\&R system design method

Note that the same information elements are used to design the system in Figure 6 as are used in Figure 5. The difference is only in the more direct application of that information in Figure 6 in the sense that surrogate metrics are no longer necessary. A limitation of the more direct approach, however, is that it requires real-time estimation of probabilities and must use reasonable probabilistic trajectory models. Probability estimation can be performed analytically or by running Monte Carlo simulations in near real time. These methods have been demonstrated in several applications, and should become even more viable as computational capabilities continue to increase $[1,2,29,30]$.

\section{IMPLEMENTATION ISSUES}

Finally, there are several other issues that are of critical importance to the operational use of a new CD\&R system. These include human performance issues in terms of the ability of the operators to respond appropriately and consistently to conflict alerts, and finding an appropriate balance between false alarms and loss-of-separation incidents. An additional issue relates to the 
integration of a new CD\&R system with other existing systems. Cases of inconsistency between air traffic controller detection and resolution of conflicts and TCAS alerts have occurred since the introduction of TCAS [31]. Additional complexity will arise as more advanced CD\&R systems are fielded on the ground and in the air. Specifically, the new systems need to be designed to operate together harmoniously within a single aircraft or ground control installation and also to work consistently between systems in different aircraft or facilities. This includes integrating CD\&R alerts with other hazard warnings (which may use different logic or detection thresholds), coordinating maneuvers between aircraft (which may be using different maneuvering dimensions), and informing operators of what is transpiring. Of special concern will be ensuring that information provided to the operator from these systems is consistent and supportive, rather than contradictory and confusing. Finally, the computational efficiency of the different CD\&R methods has not been addressed here, but would be an important consideration for implementation.

\section{CONCLUDING REMARKS}

It is clear from this survey of models that there are a large number and variety of approaches to the conflict detection and resolution problem. In regard to future approaches, however, it will be important that a larger set of issues is considered than has typically been the case in the models discussed here. These issues include the effects of uncertainty, ability to handle multiple conflicts, coordination, computational requirements, implementation issues, pilot and controller acceptance, robustness to degradation or failure, integration with other hazard information such as weather, and verification and certification requirements. The majority of the models covered here do not yet adequately address these concerns. Additionally, a consistent benchmarking method for analyzing and validating models is required. This is difficult due to the variety of operational modes and conditions to which CD\&R systems may be exposed, but will be necessary in order to select the most effective systems for implementation in the field. 
ACKNOWLEDGMENT

This research was supported by the NASA Ames Research Center under grant NAG2-1111. The authors thank many of the developers of the models reviewed here for their valuable discussions regarding conflict detection and resolution.

\section{REFERENCES}

[1] D. Isaacson and H. Erzberger, "Design of a Conflict Detection Algorithm for the Center/TRACON Automation System", in Proc. $16^{\text {th }}$ Digital Avionics Systems Conf., Irvine, CA, October 26-30, 1997, pp. 9.3-1 - 9.3-9.

[2] D. Brudnicki, K. Lindsay, and A. McFarland, “Assessment of Field Trials, Algorithmic Performance, and Benefits of the User Request Evaluation Tool (URET) Conflict Probe”, in Proc. $16^{\text {th }}$ Digital Avionics Systems Conf., Irvine, CA, October 26-30, 1997, pp. 9.3-35 9.3-44.

[3] W. E. Kelly, "Conflict Detection and Alerting for Separation Assurance Systems", in Proc. $18^{\text {th }}$ Digital Avionics Systems Conf., St. Louis, MO, October 27-29, 1999.

[4] K. Zeghal, “A Review of Different Approaches Based on Force Fields for Airborne Conflict Resolution”, AIAA-98-4240, in Proc. AIAA Guidance, Navigation, and Control Conf., Boston, MA, August 10-12, 1998, pp. 818-827.

[5] A. Warren, "Medium Term Conflict Detection for Free Routing: Operational Concepts and Requirements Analysis", in Proc. 16 $6^{\text {th }}$ Digital Avionics Systems Conf., Irvine, CA, October 26-30, 1997, pp. 9.3-27 - 9.3-34.

[6] J. Krozel, M. Peters, and G. Hunter, "Conflict Detection and Resolution for Future Air Transportation Management”, NASA CR-97-205944, April 1, 1997.

[7] J. Kuchar and L. Yang, "Survey of Conflict Detection and Resolution Modeling Methods", AIAA-97-3732, in Proc. AIAA Guidance, Navigation, and Control Conf., New Orleans, LA, August 11-13, 1997. 
[8] W. Knecht, K. Smith, and P. Hancock, “A Dynamic Conflict Probe and Index of Collision Risk", in Proc. 40 ${ }^{\text {th }}$ Annual Meeting of the Human Factors and Ergonomics Society, Philadelphia, PA, September 2-6, 1996, pp. 106-110.

[9] R. Barhydt and R. J. Hansman, "Experimental Studies of the Effect of Intent Information on Cockpit Traffic Displays", AIAA Journal of Guidance, Control, and Dynamics, vol. 22 no. 4, pp. 520-527, July-August, 1999.

[10] M. Waller and C. Scanlon, eds., "Proceedings of the NASA Workshop on Flight Deck Centered Parallel Runway Approaches in Instrument Meteorological Conditions", NASA Conf. Publication 10191, Hampton, VA, December, 1996.

[11] Radio Technical Committee on Aeronautics (RTCA), "Minimum Performance Standards Airborne Ground Proximity Warning Equipment”, Document No. RTCA/DO-161A, Washington, D.C., May 27, 1976.

[12] D. Bateman, "The Introduction of Enhanced Ground-Proximity Warning Systems (EGPWS) into Civil Aviation Operations Around the World", in Proc. $11^{\text {th }}$ Annual European Aviation Safety Seminar (EASS '99), Amsterdam, Netherlands, March 8-10, 1999.

[13] Federal Aviation Administration, "Precision Runway Monitor Demonstration Report", Document DOT/FAA/RD-91/5, February, 1991.

[14] Radio Technical Committee on Aeronautics (RTCA), "Minimum Performance Specifications for TCAS Airborne Equipment”, Document No. RTCA/DO-185, Washington, D.C., September, 1983.

[15] P. Ryan and W. Brodegard, "New Collision Avoidance Device is Based on Simple and Passive Design to Keep the Cost Low”, ICAO Journal, vol. 52 no. 4, May, 1997.

[16] F. P. Coenen, G. P. Smeaton, and A. G. Bole, "Knowledge-Based Collision Avoidance", Journal of Navigation, vol. 42 no. 1, 1989.

[17] Y. Iijima, H. Hagiwara, and H. Kasai, "Results of Collision Avoidance Maneuver Experiments Using a Knowledge-Based Autonomous Piloting System”, Journal of Navigation, vol. 44 no. 2, 1991. 
D. H. Taylor, "Uncertainty in Collision Avoidance Maneuvering”, Journal of Navigation, vol. 43 no. $2,1990$.

[19] A. Chakravarthy and D. Ghose, "Obstacle Avoidance in a Dynamic Environment - A Collision Cone Approach”, IEEE Transactions on Systems, Man, and Cybernetics, vol. 28 no. 5, pp. 562-574, September, 1998.

[20] R. Lachner, "Collision Avoidance as a Differential Game - Real-Time Approximation of Optimal Strategies Using Higher Derivatives of the Value Function”, in Proc. IEEE International Conf. on Systems, Man, and Cybernetics, Orlando, FL, October 12-15, 1997 , pp. 2308-2313.

[21] A. R. Pritchett, "Pilot Non-Conformance to Alerting System Commands During Closely Spaced Parallel Approaches", Sc.D. Thesis, Department of Aeronautics and Astronautics, Massachusetts Institute of Technology, Cambridge, MA, December, 1996.

[22] B. Carpenter and J. Kuchar, "Probability-Based Collision Alerting Logic For CloselySpaced Parallel Approach", Paper AIAA-97-0222, presented at the $35^{\text {th }}$ AIAA Aerospace Sciences Meeting and Exhibit, Reno, NV, January 6-10, 1997.

[23] V. N. Duong and E. G. Hoffman, "Conflict Resolution Advisory Service in Autonomous Aircraft Operations", in Proc. $16^{\text {th }}$ Digital Avionics Systems Conf., Irvine, CA, October 2630, 1997, pp. 9.3-10 - 9.3-17.

[24] J. Hoekstra, R. van Gent, and R. Ruigrok, "Conceptual Design of Free Flight with Airborne Separation Assurance", AIAA-98-4239, in Proc. AIAA Guidance, Navigation, and Control Conf., Boston, MA, August 10-12, 1998, pp. 807-817.

[25] K. Zeghal and E. Hoffman, "Design of Cockpit Displays for Limited Delegation of Separation Assurance", in Proc. $18^{\text {th }}$ Digital Avionics Systems Conf., St. Louis, MO, October 27-29, 1999.

[26] A. Vink, S. Kauppinen, J. Beers, and K. de Jong, "Medium Term Conflict Detection in EATCHIP Phase III", in Proc. $16^{\text {th }}$ Digital Avionics Systems Conf., Irvine, CA, October 2630, 1997, pp. 9.3-45-9.3-52. 
[27] C. Haissig, B. Corwin, and M. Jackson, "Designing an Airborne Alerting System for Closely Spaced Parallel Approaches", AIAA-99-3986, in Proc. AIAA Guidance, Navigation, and Control Conf., Portland, OR, August 9-11, 1999, pp. 280-287.

[28] M. Prandini, J. Lygeros, A. Nilim, and S. Sastry, "A Probabilistic Framework for Aircraft Conflict Detection", AIAA-99-4144, in Proc. AIAA Guidance, Navigation, and Control Conf., Portland, OR, August 9-11, 1999, pp. 1047-1057.

[29] L. Yang and J. Kuchar, "Prototype Conflict Alerting Logic for Free Flight", AIAA Journal of Guidance, Control, and Dynamics, vol. 20 no. 4, pp. 768-773, July-August, 1997.

[30] L. Yang and J. Kuchar, "Using Intent Information in Probabilistic Conflict Analysis", AIAA-98-4237, in Proc. 1998 AIAA Guidance, Navigation, and Control Conf., Boston, MA, August 10-12, 1998, pp. 797-806.

[31] V. Mellone and S. Frank, "Behavioral Impact of TCAS II on the National Air Traffic Control System", presented at the $7^{\text {th }}$ International Symposium on Aviation Psychology, Ohio State University, April 27, 1993.

[32] J. Andrews, "A Relative Motion Analysis of Horizontal Collision Avoidance”, SAFE Journal, vol. 8, no. 2, 1978.

[33] K. D. Bilimoria, H. Q. Lee, Z.-H. Mao, and E. Feron, "Comparison of Centralized and Decentralized Conflict Resolution Strategies for Multiple-Aircraft Problems”, in Proc. 2000 AIAA Guidance, Navigation, and Control Conf., Denver, CO, August, 2000.

[34] E. Frazzoli, Z.-H. Mao, J.-H. Oh, and E. Feron, "Resolution of Conflicts Involving Many Aircraft via Semidefinite Programming”, AIAA Journal of Guidance, Control, and Dynamics, in press.

[35] C. Tomlin, G. Pappas, and S. Sastry, "Conflict Resolution for Air Traffic Management: A Study in Multi-Agent Hybrid Systems”, IEEE Transactions on Automatic Control, vol. 43, no. 4, pp. 509-521, April, 1998.

[36] R. Irvine, "GEARS Conflict Resolution Algorithm”, AIAA-98-4236, in Proc. 1998 AIAA Guidance, Navigation, and Control Conf., Boston, MA, August 10-12, 1998, pp. 786-796. 
[37] T. Ota, M. Nagati, and D-C. Lee, “Aircraft Collision Avoidance Trajectory Generation”, AIAA-98-4241, in Proc. 1998 AIAA Guidance, Navigation, and Control Conf., Boston, MA, August 10-12, 1998, pp. 828-837.

[38] J. Kosecka, C. Tomlin, G. Pappas, and S. Sastry, "Generation of Conflict Resolution Maneuvers for Air Traffic Management", presented at the 1997 International Conf. on Robotics and Intelligent Systems, Sept. 1997.

[39] M. Eby, “A Self-Organizational Approach for Resolving Air Traffic Conflicts”, Lincoln Laboratory Journal, vol. 7, no. 2, 1994.

[40] M. Eby and W. Kelly, "Free Flight Separation Assurance Using Distributed Algorithms", in Proc. 1999 IEEE Aerospace Conf., Snowmass, CO, March 6-13, 1999, pp. 429-441.

[41] B. Sridhar and G. Chatterji, "Computationally Efficient Conflict Detection Methods For Air Traffic Management”, in Proc. 1997 American Control Conf., Albuquerque, NM, June 3-5, 1997.

[42] K. Havel and J. Husarcik, "A Theory of the Tactical Conflict Prediction of a Pair of Aircraft", Journal of Navigation, vol. 42, no. 3, 1989.

[43] K. Bilimoria, B. Sridhar, and G. Chatterji, "Effects of Conflict Resolution Maneuvers and Traffic Density of Free Flight", in Proc. 1996 AIAA Guidance, Navigation, and Control Conf., San Diego, CA, 1996.

[44] D. Burgess, S. Altman, and M. L. Wood, "TCAS: Maneuvering Aircraft in the Horizontal Plane", Lincoln Laboratory Journal, vol. 7, no. 2, 1994.

[45] R. Gazit, “Aircraft Surveillance and Collision Avoidance Using GPS”, PhD Thesis, Stanford University, Stanford, CA, August, 1996.

[46] K. Harper, S. Mulgund, S. Guarino, A. Mehta, and G. Zacharias, "Air Traffic Controller Agent Model for Free Flight", AIAA-99-3987, in Proc. 1999 AIAA Guidance, Navigation, and Control Conf., Portland, OR, August 9-11, 1999, pp. 288-301.

[47] W. P. Niedringhaus, "Stream Option Manager (SOM): Automated Integration of Aircraft Separation, Merging, Stream Management, and Other Air Traffic Control Functions”, IEEE 
Transactions on Systems, Man, and Cybernetics, vol. 25, no. 9, pp. 1269-1280, September, 1995.

[48] Y. Zhao and R. L. Schultz, "Deterministic Resolution of Two Aircraft Conflict in Free Flight", AIAA-97-3547, in Proc. 1997 AIAA Guidance, Navigation, and Control Conf., New Orleans, LA, August 11-13, 1997, pp. 469-478.

[49] I. Burdun and O. Parfentyev, “AI Knowledge Model for Self-Organizing Conflict

Prevention / Resolution in Close Free-Flight Air Space", in Proc. 1999 IEEE Aerospace Conf., Snowmass, CO, March 6-13, 1999, pp. 409-428.

[50] N. Durand, J. Alliot, and O. Chansou, "Optimal Resolution of En Route Conflicts”, Air

Traffic Control Quarterly, vol. 3, no. 3, pp. 139-161, 1995.

[51] R. L. Ford, "The Conflict Resolution Process for TCAS II and Some Simulation Results", Journal of Navigation, vol. 40, no. 3, 1987.

[52] J. Krozel and M. Peters, "Conflict Detection and Resolution for Free Flight", Air Traffic Control Quarterly, vol. 5, no. 3, pp. 181-212, 1997.

[53] D. Love, "TCAS III: Bringing Operational Compatibility to Airborne Collision Avoidance", presented at the $8^{\text {th }}$ Digital Avionics Systems Conf., 1988.

[54] P. K. Menon, G. D. Sweriduk, and B. Sridhar, "Optimal Strategies for Free-Flight Air Traffic Conflict Resolution", AIAA Journal of Guidance, Control, and Dynamics, vol. 22, no. 2, pp. 202-211, March-April, 1999.

[55] W. P. Niedringhaus, "Maneuver Option Manager: Automated Simplification of Complex Air Traffic Control Problems", IEEE Transactions on Systems, Man, and Cybernetics, vol. 22, no. 5, pp. 1047-1057, September-October, 1992.

[56] R. Schild, "Rule Optimization for Airborne Aircraft Separation", PhD Thesis, Technical University of Vienna, Austria, November 26, 1998.

[57] K. Zeghal, "Towards the Logic of an Airborne Collision Avoidance System which Ensures Coordination with Multiple Cooperative Intruders", ICAS-94-8.6.4, ICAS Proceedings, vol. 3, pp. 2208-2218, 1994. 
[58] R. L. Ford and D. L. Powell, “A New Threat Detection Criterion for Airborne Collision Avoidance Systems", Journal of Navigation, vol. 43, no. 3, 1990.

[59] S. Ratcliffe, "Automatic Conflict Detection Logic for Future Air Traffic Control”, Journal of Navigation, vol. 42, no. 3, 1989.

[60] T. Shepard, T. Dean, W. Powley, and Y. Akl, "A Conflict Prediction Algorithm Using Intent Information", in Proc. 36 ${ }^{\text {th }}$ Annual Air Traffic Control Assoc. Conf., Fall 1991.

[61] J. M. Shewchun, J-H. Oh, and E. Feron, "Linear Matrix Inequalities for Free Flight Conflict Problems", in Proc. IEEE Conf. on Decision and Control, San Diego, CA, December, 1997, pp. 2417-2422.

[62] R. Paielli and H. Erzberger, "Conflict Probability Estimation for Free Flight", Journal of Guidance, Control, and Dynamics, vol. 20 no. 3, May-June, 1997.

[63] G. Bakker and H. Blom, “Air Traffic Collision Risk Modeling”, in Proc. 32 ${ }^{\text {nd }}$ IEEE Conf. on Decision and Control, vol. 2, San Antonio, TX, December, 1993.

[64] J. P. Wangermann and R. F. Stengel, "Principled Negotiation Between Intelligent Agents: A Model for Air Traffic Management”, ICAS-94-8.6.3, ICAS Proceedings, vol. 3, pp. 21972207, 1994.

[65] J. P. Wangermann and R. F. Stengel, "Optimization and Coordination of Multi-Agent Systems Using Principled Negotiation", AIAA-96-3853, in Proc. 1996 AIAA Guidance, Navigation, and Control Conf., San Diego, CA, July 29-31, 1996.

[66] M. Innocenti, P. Gelosi, and L. Pollini, “Air Traffic Management using Probability Function Fields", AIAA-99-4149, in Proc. 1999 AIAA Guidance, Navigation, and Control Conf., Portland, OR, August 9-11, 1999, pp. 1088-1097.

[67] H. Rome and R. Kalafus, "Impact of Automatic Dependent Surveillance and Navigation System Accuracy on Collision Risk on Intersecting Tracks", in Proc. National Technical Meeting of the Institute of Navigation, 1988.

[68] P. R. Williams, "Aircraft Collision Avoidance using Statistical Decision Theory", in Proc. Sensors and Sensor Systems for Guidance and Navigation II, 1992. 
[69] G. Heuvelink and H. Blom, “An Alternative Method to Solve a Variational Inequality Applied to an Air Traffic Control Example", presented at the Analysis and Optimization of Systems: $8^{\text {th }}$ International Conf., 1988.

[70] H. von Viebahn and J. Schiefele, "A Method for Detecting and Avoiding Flight Hazards", in Proc. SPIE Meeting on Enhanced and Synthetic Vision, Bellingham, WA, April 21-22, 1997, pp. 50-56.

[71] B. McNally, R. Bach, and W. Chan, "Field Test Evaluation of the CTAS Conflict Prediction and Trial Planning Capability”, AIAA-98-4480, in Proc. 1998 AIAA Guidance, Navigation, and Control Conf., Boston, MA, August 10-12, 1998, pp. 1686-1697.

[72] D. Brudnicki and A. McFarland, "User Request Evaluation Tool (URET) Conflict Probe Performance and Benefits Assessment”, Report MP97W0000112, MITRE Corp., June, 1997. 
Table 1: Principal Modeling Methods: Nominal Trajectory Propagation Model Dimensions Detection Resolution Maneuvers Multiple

\begin{tabular}{lccccc}
\hline \hline Andrews [32] & $\mathrm{H}$ & - & $\mathrm{O}$ & $\mathrm{T}$ & $\mathrm{P}$ \\
Bilimoria [33] & $\mathrm{H}$ & - & $\mathrm{O}$ & $\mathrm{C}(\mathrm{ST})$ & $\mathrm{P} \& \mathrm{G}$ \\
Chakravarthy [19] & $\mathrm{H}$ & - & $\mathrm{O}$ & $\mathrm{C}(\mathrm{ST})$ & $\mathrm{P}$ \\
Frazzoli [34] & $\mathrm{H}$ & - & $\mathrm{O}$ & $\mathrm{C}(\mathrm{ST})$ & $\mathrm{G}$ \\
Tomlin [35] & $\mathrm{H}$ & - & $\mathrm{O}$ & $\mathrm{T}$ & $\mathrm{G}$ \\
Irvine [36] & $\mathrm{HV}$ & - & $\mathrm{O}$ & $\mathrm{C}(\mathrm{STV})$ & $\mathrm{P}$ \\
Ota [37] & $\mathrm{HV}$ & - & $\mathrm{O}$ & $\mathrm{C}(\mathrm{TV})$ & $\mathrm{G}$ \\
Kosecka [38] & $\mathrm{H}$ & - & $\mathrm{F}$ & $\mathrm{C}(\mathrm{ST})$ & $\mathrm{G}$ \\
Zeghal [4] & $\mathrm{H}$ & - & $\mathrm{F}$ & $\mathrm{C}(\mathrm{ST})$ & $\mathrm{G}$ \\
Eby [39,40] & $\mathrm{HV}$ & - & $\mathrm{F}$ & $\mathrm{C}(\mathrm{STV})$ & $\mathrm{G}$ \\
Sridhar [41] & $\mathrm{H}$ & $\sqrt{ }$ & - & - & $\mathrm{P}$ \\
EGPWS [12] & $\mathrm{HV}$ & $\sqrt{ }$ & - & - & $\mathrm{P}$ \\
Havel [42] & $\mathrm{HV}$ & $\sqrt{ }$ & - & - & $\mathrm{P}$ \\
Kelly [3] & $\mathrm{HV}$ & $\sqrt{ }$ & - & - & $\mathrm{P}$ \\
TCAD [15] & $\mathrm{HV}$ & $\sqrt{ }$ & - & - & $\mathrm{P}$ \\
GPWS [11] & $\mathrm{V}$ & $\sqrt{ }$ & $\mathrm{P}$ & $\mathrm{V}$ & $\mathrm{P}$ \\
PRM [13] & $\mathrm{H}$ & $\sqrt{ }$ & $\mathrm{P}$ & $\mathrm{C}(\mathrm{TV})$ & $\mathrm{P}$ \\
Bilimoria [43] & $\mathrm{HV}$ & $\sqrt{ }$ & $\mathrm{P}$ & $\mathrm{STV}$ & $\mathrm{P}$ \\
Burgess [44] & $\mathrm{H}$ & $\sqrt{ }$ & $\mathrm{O}$ & $\mathrm{TV}$ & $\mathrm{P}$ \\
Coenen [16] & $\mathrm{H}$ & $\sqrt{ }$ & $\mathrm{O}$ & $\mathrm{ST}$ & $\mathrm{P}$ \\
Gazit [45] & $\mathrm{H}$ & $\sqrt{ }$ & $\mathrm{O}$ & $\mathrm{VT}$ & $\mathrm{P}$ \\
Harper [46] & $\mathrm{H}$ & $\sqrt{ }$ & $\mathrm{O}$ & $\mathrm{C}(\mathrm{ST})$ & $\mathrm{G}$ \\
Iijima [17] & $\mathrm{H}$ & $\sqrt{ }$ & $\mathrm{O}$ & $\mathrm{ST}$ & $\mathrm{P}$ \\
Niedringhaus [47] & $\mathrm{H}$ & $\sqrt{ }$ & $\mathrm{O}$ & $\mathrm{C}(\mathrm{ST})$ & $\mathrm{G}$ \\
Zhao [48] & $\mathrm{H}$ & $\sqrt{ }$ & $\mathrm{O}$ & $\mathrm{T}$ & $\mathrm{P}$ \\
Burdun [49] & $\mathrm{HV}$ & $\sqrt{ }$ & $\mathrm{O}$ & $\mathrm{C}(\mathrm{STV})$ & $\mathrm{P}$ \\
Durand [50] & $\mathrm{HV}$ & $\sqrt{ }$ & $\mathrm{O}$ & $\mathrm{T}$ & $\mathrm{G}$ \\
Ford [51] & $\mathrm{HV}$ & $\sqrt{ }$ & $\mathrm{O}$ & $\mathrm{V}$ & $\mathrm{P}$ \\
Krozel [6,52] & $\mathrm{HV}$ & $\sqrt{ }$ & $\mathrm{O}$ & $\mathrm{STV}$ & $\mathrm{P}$ \\
Love [53] & $\mathrm{HV}$ & $\sqrt{ }$ & $\mathrm{O}$ & $\mathrm{TV}$ & $\mathrm{P}$ \\
Menon [54] & $\mathrm{HV}$ & $\sqrt{ }$ & $\mathrm{O}$ & $\mathrm{C}(\mathrm{STV})$ & $\mathrm{G}$ \\
Niedringhaus [55] & $\mathrm{HV}$ & $\sqrt{ }$ & $\mathrm{O}$ & $\mathrm{STV}$ & $\mathrm{G}$ \\
Schild [56] & $\mathrm{HV}$ & $\sqrt{ }$ & $\mathrm{O}$ & $\mathrm{C}(\mathrm{TV})$ & $\mathrm{P}$ \\
TCAS [14] & $\mathrm{HV}$ & $\sqrt{ }$ & $\mathrm{O}$ & $\mathrm{V}$ & $\mathrm{P}$ \\
Hoekstra [24] & $\mathrm{HV}$ & $\sqrt{ }$ & $\mathrm{F}$ & $\mathrm{C}(\mathrm{STV})$ & $\mathrm{P}$ \\
Zeghal [57] & $\mathrm{HV}$ & $\sqrt{ }$ & $\mathrm{F}$ & $\mathrm{C}(\mathrm{STV})$ & $\mathrm{G}$ \\
Duong [23] & $\mathrm{V}$ & $\mathrm{M} / \mathrm{F}$ & $\mathrm{C}(\mathrm{STV})$ & $\mathrm{P}$ \\
\hline \hline
\end{tabular}


Table 2: Principal Modeling Methods: Worst-Case Trajectory Propagation Model Dimensions Detection Resolution Maneuvers Multiple

\begin{tabular}{|c|c|c|c|c|c|}
\hline Lachner [20] & $\mathrm{H}$ & - & $\mathrm{O}$ & $\mathrm{C}(\mathrm{ST})$ & $\mathrm{P}$ \\
\hline Tomlin [33] & $\mathrm{H}$ & - & $\mathrm{O}$ & $\mathrm{S}$ & $\mathrm{G}$ \\
\hline Ford [58] & $\mathrm{H}$ & $\sqrt{ }$ & - & - & $\mathrm{P}$ \\
\hline Ratcliffe [59] & $\mathrm{HV}$ & $\sqrt{ }$ & - & - & $\mathrm{P}$ \\
\hline Shepard [60] & $\mathrm{HV}$ & $\sqrt{ }$ & - & 一 & $\mathrm{P}$ \\
\hline Shewchun [61] & $\mathrm{HV}$ & $\sqrt{ }$ & - & - & $\mathrm{P}$ \\
\hline AILS [10] & $\mathrm{HV}$ & $\sqrt{ }$ & $\mathrm{P}$ & $\mathrm{C}(\mathrm{TV})$ & $\mathrm{P}$ \\
\hline Gazit [43] & $\mathrm{H}$ & $\sqrt{ }$ & $\mathrm{O}$ & VT & $\mathrm{P}$ \\
\hline Vink [26] & $\mathrm{HV}$ & $\sqrt{ }$ & M & $\mathrm{C}(\mathrm{STV})$ & $\mathrm{P}$ \\
\hline
\end{tabular}


Table 3: Principal Modeling Methods: Probabilistic Trajectory Propagation

\begin{tabular}{|c|c|c|c|c|c|}
\hline Model & Dimensions & Detection & Resolution & Maneuvers & Multiple \\
\hline Paielli [62] & $\mathrm{H}$ & - & - & - & $\mathrm{P}$ \\
\hline Taylor [18] & $\mathrm{H}$ & - & - & - & $\mathrm{P}$ \\
\hline Bakker [63] & $\mathrm{HV}$ & - & - & - & $\mathrm{P}$ \\
\hline Wangermann $[64,65]$ & $\mathrm{HV}$ & - & $\mathrm{O}$ & $\mathrm{C}(\mathrm{STV})$ & $\mathrm{G}$ \\
\hline Innocenti [66] & $\mathrm{H}$ & - & $\mathrm{F}$ & $\mathrm{C}(\mathrm{ST})$ & G \\
\hline Rome [67] & $\mathrm{H}$ & $\sqrt{ }$ & - & - & $\mathrm{P}$ \\
\hline Warren [5] & $\mathrm{H}$ & $\sqrt{ }$ & - & - & $\mathrm{P}$ \\
\hline Williams [68] & $\mathrm{HV}$ & $\sqrt{ }$ & - & - & $\mathrm{P}$ \\
\hline Carpenter [22] & $\mathrm{H}$ & $\sqrt{ }$ & $\mathrm{P}$ & $\mathrm{C}(\mathrm{TV})$ & $\mathrm{P}$ \\
\hline Heuvelink [69] & $\mathrm{H}$ & $\sqrt{ }$ & $\mathrm{O}$ & $\mathrm{S}$ & $\mathrm{P}$ \\
\hline Prandini [28] & $\mathrm{H}$ & $\sqrt{ }$ & $\mathrm{O}$ & $\mathrm{T}$ & $\mathrm{P}$ \\
\hline Krozel [6] & $\mathrm{HV}$ & $\sqrt{ }$ & $\mathrm{O}$ & STV & $\mathrm{P}$ \\
\hline von Viebahn [70] & $\mathrm{HV}$ & $\sqrt{ }$ & $\mathrm{O}$ & TV & $\mathrm{P}$ \\
\hline CTAS $[1,71]$ & $\mathrm{HV}$ & $\sqrt{ }$ & $\mathrm{M}$ & $\mathrm{C}(\mathrm{STV})$ & $\mathrm{P}$ \\
\hline URET $[2,72]$ & $\mathrm{HV}$ & $\sqrt{ }$ & M & $\mathrm{C}(\mathrm{STV})$ & $\mathrm{P}$ \\
\hline Yang $[29,30]$ & $\mathrm{HV}$ & $\sqrt{ }$ & $\mathrm{M}$ & $\mathrm{C}(\mathrm{STV})$ & $\mathrm{P}$ \\
\hline
\end{tabular}


Table 4: Model Categorization AbBReViations

Column in Tables 1-3

Abbreviations

\begin{tabular}{|c|c|}
\hline \multirow[t]{3}{*}{ Dimensions } & $\mathrm{H}=$ Horizontal plane only \\
\hline & $\mathrm{V}=$ Vertical plane only \\
\hline & $\mathrm{HV}=$ Horizontal and Vertical planes \\
\hline \multirow[t]{2}{*}{ Detection } & $\sqrt{ }=$ Explicit conflict detection threshold \\
\hline & $-=$ No explicit conflict detection threshold \\
\hline \multirow[t]{5}{*}{ Resolution } & $\mathrm{P}=$ Prescribed \\
\hline & $\mathrm{O}=$ Optimized \\
\hline & $\mathrm{F}=$ Force Field \\
\hline & M = Manual \\
\hline & $-=$ Resolution maneuvers not considered \\
\hline \multirow[t]{4}{*}{ Maneuvers } & $\mathrm{T}=$ Turns \\
\hline & $\mathrm{V}=$ Vertical maneuvers \\
\hline & $\mathrm{S}=$ Speed changes \\
\hline & $\mathrm{C}()=$ Combined $/$ simultaneous maneuvers \\
\hline \multirow[t]{2}{*}{ Multiple } & $\mathrm{P}=$ Pairwise \\
\hline & $\mathrm{G}=$ Global \\
\hline
\end{tabular}

